\title{
Risk-Adjusted Cancer Screening and Prevention (RiskAP): Complementing Screening for Early Disease Detection by a Learning Screening Based on Risk Factors
}

\author{
Rita K. Schmutzler ${ }^{a}$ Björn Schmitz-Luhn ${ }^{b}$ Bettina Borisch ${ }^{c}$ Peter Devilee ${ }^{d}$ \\ Diana Eccles $^{e}$ Per Hall ${ }^{f}$ Judith Balmaña ${ }^{g}$ Stefania Bocciah, ${ }^{\text {i }}$ Peter Dabrock ${ }^{j}$ \\ Günter Emons $^{k}$ Wolfgang Gaissmaier ${ }^{l}$ Jacek Gronwald ${ }^{m}$ Stefanie Houwaart ${ }^{n}$ \\ Stefan Huster ${ }^{\circ}$ Karin Kast ${ }^{a}$ Alexander Katalinic ${ }^{p}$ Sabine C. Linn ${ }^{q}$ \\ Sowmiya Moorthie ${ }^{r}$ Paul Pharoah $^{\mathrm{s}}$ Kerstin Rhiem ${ }^{\mathrm{a}}$ Tade Spranger $^{\mathrm{t}}$ \\ Dominique Stoppa-Lyonnet ${ }^{\mathrm{u}}$ Johannes Jozef Marten van Delden ${ }^{\mathrm{v}}$ \\ Marc van den Bulckew Christiane Woopen ${ }^{\mathrm{b}}$ \\ ${ }^{a}$ Center Familial Breast and Ovarian Cancer and Center of Integrated Oncology (CIO), University Hospital Cologne,

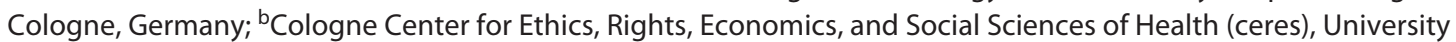 \\ of Cologne, and Research Unit Ethics, University Hospital of Cologne, Cologne, Germany; 'Institute of Global Health, \\ University of Geneva, Geneva, Switzerland; 'Leids Universitair Medisch Zentrum, Universiteit Leiden, Leiden, The \\ Netherlands; ${ }^{\mathrm{e} C l i n i c a l}$ Trials Unit, University of Southampton, Southampton, UK; ${ }^{\circ}$ Karolinska Institutet, Stockholm, \\ Sweden; ${ }^{9}$ Vall d'Hebron Instituto de Oncologia (VHIO), Barcelona, Spain; ${ }^{\text {h}}$ Sezione di Igiene, Instituto di Sanità \\ Pubblica, Università Cattolica del Sacro Cuore, Rome, Italy; 'Department of Woman and Child Health and Public \\ Health - Public Health Area, Fondazione Policlinico Universitario A. Gemelli IRCCS, Rome, Italy; ${ }^{\text {}}$ Friedrich-Alexander- \\ Universität, Erlangen, Germany; kUniklinik Göttingen, Georg-August-Universität Göttingen, Göttingen, Germany; \\ 'Max-Planck-Institut für Bildungsforschung, Universität Konstanz, Konstanz, Germany; 'mInternational Hereditary \\ Cancer Center, Department of Genetics and Pathology, Pomeranian Medical University, Szczecin, Poland; "BRCA- \\ Netzwerk, Bonn, Germany; 'Lehrstuhl für Öffentliches Recht, Sozial- und Gesundheitsrecht und Rechtsphilosophie, \\ Ruhr-Universität Bochum, Bochum, Germany; PInstitut für Krebsepidemiologie, Universität Lübeck, Lübeck, \\ Germany; ${ }^{9}$ Departments of Medical Oncology and Molecular Pathology - Netherlands Cancer Institute, Amsterdam, \\ The Netherlands; 'PHG Foundation, University of Cambridge, Cambridge, UK; ${ }^{\text {DDepartment }}$ of Oncology, University

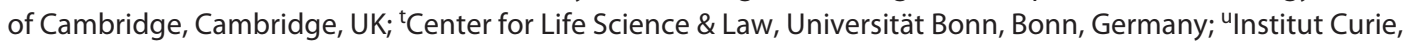 \\ Paris, France; vUM Utrecht - Julius Center for Health Sciences and Primary Care, Universiteit Utrecht, Utrecht, The \\ Netherlands; "'Belgium Cancer Center (BCC), Brussels, Belgium
}

\section{Keywords}

Risk-adjusted prevention - Breast cancer - Evidencegenerating care $\cdot$ ELSI ethical, legal, social implications

\footnotetext{
Abstract

Background: Risk-adjusted cancer screening and prevention is a promising and continuously emerging option for improving cancer prevention. It is driven by increasing
}

knowledge of risk factors and the ability to determine them for individual risk prediction. However, there is a knowledge gap between evidence of increased risk and evidence of the effectiveness and efficiency of clinical preventive interventions based on increased risk. This gap is, in particular, aggravated by the extensive availability of genetic risk factor diagnostics, since the question of appropriate preventive

Rita K. Schmutzler and Björn Schmitz-Luhn are co-first authors.

\section{karger@karger.com} www.karger.com/brc

\section{Karger $\%$}

BOPEN ACCESS
(C) 2021 The Author(s)

Published by S. Karger AG, Basel

This is an Open Access article licensed under the Creative Common Attribution-NonCommercial-4.0 International License (CC BY-NC) (http://www.karger.com/Services/OpenAccessLicense), applicable to the online version of the article only. Usage and distribution for commercial purposes requires written permission.
Correspondence to:

Rita K. Schmutzler, rita.schmutzler@uk-koeln.de 
measures immediately arises when an increased risk is identified. However, collecting proof of effective preventive measures, ideally by prospective randomized preventive studies, typically requires very long periods of time, while the knowledge about an increased risk immediately creates a high demand for action. Summary: Therefore, we propose a riskadjusted prevention concept that is based on the best current evidence making needed and appropriate preventive measures available, and which is constantly evaluated through outcome evaluation, and continuously improved based on these results. We further discuss the structural and procedural requirements as well as legal and socioeconomical aspects relevant for the implementation of this concept.

(C) 2021 The Author(s).

Published by S. Karger AG, Basel

\section{Executive Summary}

Cancer screening has been introduced in many Western countries, but its effectiveness remains subject of debate, particularly now that new possibilities to predict cancer risk are becoming available. These are driven forward by high-throughput "multi-omics" technologies comprising, among others, genomics, transcriptomics, and proteomics, which have led to the discovery of new molecular risk factors that seem to interact with each other and with non-genetic risk factors in a multiplicative manner. Personalized risk prediction by genome-based knowledge and technology opens up new opportunities for increasingly individual-oriented risk-adjusted cancer prevention. Consumer-oriented information systems such as health-related apps and algorithms are already profoundly changing health care services. The convergence of such innovative information and biotechnology systems enables the dissemination of risk prediction models that will reinvent the way in which health care providers interact with individuals at risk for certain diseases.

Heritability of cancer overall has been estimated at around $33 \%$, significantly so for skin melanoma, prostate, ovary, breast, and several other cancers [1-3]. For breast cancer, approximately half of the familial risk has been deciphered, and for this reason it has been the leading use case of this insight in the field of cancer prevention. Based on its genetic make-up, breast cancer can be considered as multiple rare diseases, which are influenced by different lifestyle and environmental factors. Genetic and interacting non-genetic risk factors can also be used to predict future risks in healthy relatives of women affected by breast cancer. This use case will be therefore serving in this paper to illustrate and exemplify the state of the art and the current challenges in cancer prediction.

A variety of genetic tests for predicting the risk of breast cancer are already available on the health market, sometimes fueling an expectation to determine the specific risk for developing cancer in any given person solely on these grounds. These genetic tests are used as part of complex algorithms to determine a potentially increased risk of disease, and patients and doctors are increasingly using such tests. However, the ability to categorize risk in this way has advanced more rapidly than the development of evidence regarding the clinical utility for preventive measures. The development of comprehensive genetic and risk literacy of doctors and affected persons has been lagging behind, contributing to an often-uninformed assessment of benefits and harms associated with preventive measures. This, in turn, can lead to ill-informed management choices, potentially causing harm through unnecessary medical interventions and generating unnecessary expenses. For this reason, in a general population screening, specific clinical measures based on the sole risk prediction through genetic testing are not justified, as has been outlined by public health groups [46]. On the other hand, ignoring the potential for genetic testing to improve the benefit/harm ratio for patients and populations may impede the creation of effective strategies to improve current approaches to screening and prevention.

Introducing predictive genetic testing and risk assessment into breast cancer population screening programs in order to improve clinical care and impact on prevention will disrupt current practice and require a continuous balancing of rigorous outcome evaluation and timely adaptation of the health care system. Therefore, we propose a multi-step translational concept, which allows health care systems to meet the current demand for genetic testing while capturing evidence about its clinical utility at the same time. Specifically, the offer of risk-predictive testing should be integrated into an evidence- or knowledge-generating care concept, allowing for safe and quality-controlled use of genetic testing in a clinical setting coupled with consistent recording of costs and interventions over time, impact on overall and cancer-free survival and including patient-reported outcomes around quality of life. This extended framework of data collection, eased by the newly available digital solutions for data collection, may facilitate the move towards a learning health system that allows the use of state-of-the-art technology in clinical care and at the same time complements evidence-based medicine. Also, clinical guidelines can be continuously monitored for concordance with intended patient outcome, and adapted if deemed necessary.

Key components for delivery will be translational, comprehensive care centers that are highly specialized in genomic and risk prediction medicine. They should build networks with cancer centers and primary care practitioners. Jointly, they will deliver digitized risk estimations and risk-adjusted preventive measures based on risk fac- 
tor-driven, quality-assured, and adaptable risk prediction models. They will also define common entry points for administering such risk-assessment, e.g., on the occasion of existing health screening programs for the general population. Such a cross-sectoral care concept will enable the implementation of accepted outcome measures and their connection to data collected in existing and additionally established cancer registries, to ensure long-term followup of uptakers of screening with respect to hard endpoints such as mortality, morbidity, and quality of life. This, in turn, will allow for adjustment of the care concept within an iterative knowledge-generating cycle of care. This concept, developed specifically for breast cancer, may serve as a template for other applications of genomedriven medicine such as other hereditary tumor syndromes, in personalized as well as in targeted therapeutic strategies.

\section{Introduction}

Cancer screening programs have been in place in many countries. So far, existing screening programs focus on the early diagnosis of specific diseases, e.g., by way of mammography, or the highly specific search for diseasecausing factors, like HPV infection according to well-established screening criteria [7]. Despite an ever-increasing catalogue of known risk factors for the development of cancers, the selection of the target population for existing screening programs is largely based on age and gender. However, a simple strategy for defining a target population, while administratively pragmatic, is not necessarily the optimal solution for best value, also from a health economic or a health improvement perspective. There are disadvantages of population-based screening in which many individuals are invited into a screening program despite being at low personal risk. These include stress and anxiety from the screening intervention itself, waiting for results, and from confirmatory investigation of false positive or inconclusive results requiring unnecessary additional medical interventions. Another problem of age-based population-screening is that it fails to include younger individuals already at risk levels exceeding those defined to enter the screening program, e.g., women with a BRCA1 or BRCA2 mutation who can develop breast cancer much earlier than the defined age of the screening program [8]. Finally, the screening interval and methodology that is effective for an age-based population may be inappropriate for a population at particularly high risk. E.g., even mammograms starting at age 40 would fail to detect around half the cases of breast cancer in $B R C A 1$ gene carriers: these have a median age at onset of 42 years - thus, almost half the cases which occur under this age would not be detected.
New knowledge about genetic and non-genetic risk factors, genetic testing, and the "omics" revolution are leading to a constantly evolving understanding of risk profiles. It therefore seems reasonable to put to use the already existing wealth of knowledge about the multitude of other risk factors besides age and gender and offer riskadjusted screenings using multi-factor risk-prediction models [6,9-12]. It should be noted at this point that the distinguishment between risk factors and indicators, e.g., according to the Bradford-Hill criteria, becomes increasingly blurred the more complex the risk determination for a disease becomes. The prevailing understanding seems to be that risk indicators are correlated with the disease, while risk factors are causal for the disease. However, causality is difficult to prove in complex diseases with incomplete penetrance whose pathogenesis is based on an interaction of many factors. Furthermore, the correlation of many low-risk gene variants with tumorigenesis and the multiplicative interaction of these variants has been shown, their function or correlation with a causal variant has not yet been established. This holds true for both non-genetic and genetic risk factors. Therefore, in this paper, both factors and indicators will be simply denoted as "factors."

Conceptual frameworks have been developed to address the key issues and challenges of risk-adjusted screening [13-16]. A streamlined intervention program could consider individual risks, including both genetic and non-genetic ones, e.g., family history, lifestyle, and many more, and should be complemented by a well-designed approach to monitoring outcomes. These would not only include survival but also patient-reported outcomes and health care costs allowing future analyses and iterative redesign of the program to improve the benefits and minimize the risks.

With increasing awareness and the marketing approach by a multitude of biotech companies, there is a growing implementation gap between what is technologically possible and what is available - or refundable by insurances or health care schemes - in practice [17]. Therefore, people are increasingly accessing private options for genetic testing known as "direct to consumer tests" (DTC), whose availability is accelerated by laboratories having an incentive to introduce and offer new genetic tests at an astounding rate [18]. These private options are not always well regulated and do not collect outcome data - posing a challenge for safeguarding scientific quality and not documenting or even taking into account clinical utility [19]. This leads to a "data drain" from the clinical-scientific towards the commercial sector at a time when data sharing and data mining should enable reliable, evaluated, and high-quality clinical data which is ever more vital for improving health care in a responsible way. 
The investigation of causal factors and model calibration in less common subtypes of disease, as, i.e., knowledge about the genetic factors of subtypes becomes more and more differentiated, in turn requires data collections of a size hitherto unavailable.

Because of its potential to revolutionize or disrupt conventional medicine, genome-based health information and technologies (GBHIT) have attracted the attention of health policy makers throughout Europe. In the recently launched innovative Partnership for Action Against Cancer (iPAAC) Joint Action (JA), whose main objective is to implement innovative approaches to cancer control, one of the top priorities is to integrate genomics in the health care system (www.ipaac.eu). The current initiative takes up on the groundwork of the Public Health Genomics European Network (PHGEN) under the EU health program, which has provided a best practice guideline for quality assurance, provision, and use of GBHIT following the public health trias, i.e., assessment, policy development, and assurance (http://www.phgen.eu)/, in their "Declaration of Rome" from 2012 [5]. Priority setting of the PHGEN comprises, among others, the improvement of genetic literacy and knowledge transfer by the provision of education programs and the involvement of electronic and mass media, the investment in dedicated infrastructures and databases, and the stimulation of research to produce evidence for clinical utility as well as cost-effectiveness. Moreover, it seems desirable that public health assessment should also take into account personal utility given the uniqueness of each individual genome, and beyond inter-individual clinical utility $[5,20]$. While demonstration of clinical utility is considered a prerequisite for clinical translation, the challenge is how to deal with the trade-off between the available evidence and timing the introduction of GBHIT since the evaluation of clinical utility is often lagging behind the market launch of genetic tests.

For adopting new health care options, including any new screening program, prospective randomized studies are considered gold standard in the hierarchy of evidence. In this respect, a risk-adjusted surveillance strategy could be compared to current standard population screening in a cluster randomized trial. However, such a trial would need to involve a very large population base, potentially be multi-national, and may raise insurmountable ethical and practical barriers to a successful conclusion.

To close this gap, it should be possible to collect data that demonstrate clinical utility whilst already integrating genome-based selection tests for entry to clinical screening and care [21]. This could be done by way of a multistep evaluation of clinical utility, thus creating evidence and benefit at the same time, by complementing traditional evidence-based evaluation with evidence-generating clinical care. One option within this context is the "coverage with evidence development" (CED) approach which provides provisional access to novel medical interventions while the evidence needed to assess the value of an intervention, and consequently to make coverage unconditional, is generated (cf., elaborating chances and disadvantages of this approach with specific respect to the German regulatory situation: [22]). CED - in some way or form - has already been implemented in many countries throughout the world, usually as part of an established policy framework. In consequence, it is also known under various terms such as "interim funding," "only in research (OIR)," "still in clinical research," and "conditionally funded field evaluation (CFFE)." Following such an approach would generally accommodate the rising demand of patients and doctors to use the array of available GBHIT applications, and ensure that the testing is quality-assured and the outcomes are carefully collected and collated. At the same time, clinical outcomes can be assessed confirming whether (a) specific genetic alterations are associated with increased disease risk, (b) genetic variants are indicative of the presence of specific clinical criteria and a predictable disease course, and (c) the application of this approach to cancer screening leads to clinical interventions with improved outcome, i.e., reduction of morbidity and mortality and/or increase in quality of life.

This proposed approach would allow for potentially more effective screening than currently offered. Adjusting screening to fit individual risk profiles should minimize harmful effects and maximize the benefits of screening. At the same time, the generation of new medical knowledge about risk factors and their influence on disease development and prognosis could be captured for ongoing research into clinical applications of the new genomic data.

If knowledge-based conventional screening can be complemented by knowledge-generating risk-adjusted screening, it can ensure that consumers have structured and equal access to such genetically driven risk predictions as well as clinical programs based on them $[23,24]$. Nevertheless, this concept requires the formation of cross-sectoral networks between highly specialized units and health care providers to guarantee high-quality genetic testing and clinical interpretation. It also needs to be accompanied by communication and teaching programs in order to facilitate knowledge transfer from specialized centers to primary providers and to improve genetic and risk literacy of consumers [25-28]. Finally, the generation of high-quality clinical evidence about genetic tests must still be pursued by the best available standards - e.g., by large-scale double-blind controlled clinical trials. By putting the new knowledge to work in the meantime, however, evidence can also be generated within their clinical use and fed back into the chain of knowledge generation. Prospective controlled cohort 
studies including control groups in combination with registries as prerequisites for outcomes research are considered the optimal setting for these highly translational care concepts thus enabling a dynamic and iterative bench-to-bedside and bedside-to-bench translational continuum [29-31]. In the following, the concept is outlined in more detail.

\section{Risk Model Development through a Multi-Step Learning Screening for Breast Cancer: The Concept}

While established screening programs aim at the identification of early disease stages, and use screening to grasp the widest-possible part of the population, any screening can these days become increasingly individualized, based on genetic and other factors known to indicate a specifically high (or low) risk.

Current scientific findings on breast cancer suggest that risk-adjusted prevention based on comprehensive risk assessment considering genetic and non-genetic risk factors may be more effective with respect to clinical outcome and participation rates than existing breast screening programs that offer mammography screening to the general population based on a certain age range.

In general, screening programs attempt to identify occult but already manifest cancers in an early state, allowing for curative treatment and thus better prognosis. Their utility is based on the identification of early stages of disease, ideally before they become noticeable to the individual. Beyond that, risk-adjusted screening seeks to identify and detect, in addition to mere age, individual risks before, and notwithstanding, the detection of early disease stages. Risk-adjusted screening thus comprises both individual risk assessment and early detection based on the outcome of that assessment. By exploiting all known and available risk factor information of an individual, as opposed to a single criterion like age, a personalized entry into the screening program becomes possible. Women who reach the risk threshold at earlier ages than the current entry age can, for example, largely benefit from screening, whereas for women who do not reach that threshold, side effects and costs can be diminished with a low risk of missing any cancer events. Early detection of breast cancer therefore becomes merely a part of an integrative screening program adapted to individual risk profiles, in which the focus lies not on early detection but on risk management from the onset, incorporating methods of risk detection as needed, but not being limited to them. Specifically, a cascade system of diagnostic measures should be streamlined (a) with the available knowledge on genetic and other risk factors, and (b) with the individual risk of the person at stake.
In a multi-step risk-adjusted learning screening program, risk factors are individually tested first, and with regard to the general population. For breast cancer, validated genetic risk factors exist with respect to mutation prevalence rates in the BRCA1/2 genes [32-34]. Persons positive for certain risk factors (including, as the case lies with current programs, age and gender, but also a variety of other known risk factors such as family history, mutations in risk genes, and breast density) are then subjected to the second screening phase which would include a more scrutinized risk assessment, e.g., by the calculation of a comprehensive risk score including, beyond the other risk factors, genetic testing for high, moderate, and low risks and their assessment by algorithms, identifying particular high risks by low-invasive means. As a third step, measures for early detection, e.g., intensified early diagnosis and monitoring, are offered in accordance with the individual risk identified in the first two steps. For example, when a person is found to have an average risk, the current screening offers would remain unchanged. Persons with a low risk could be offered less intensive, and persons with an increased risk more comprehensive early detection screening.

In order to identify persons or groups with particularly high or low risk to be offered a cascading risk assessment, diagnosis, and risk-based screening, existing health screening programs can be complemented by a multistep risk-adjusted learning screening system that includes genetic information and other risk factors. Naturally, the appropriate time and entrance point as well as the combination with existing health checkup or cancer screening programs should be made according to the penetrance of the respective disease. As a starting point, women in existing breast cancer mammography screenings could be additionally offered genetic analysis and pertinent non-genetic risk-factor anamnesis according to current knowledge on their impact on disease risk and offered participation in risk-adjusted structured screening programs. However, importantly, there needs to be a minimum standard of evidence supporting the declaration of a risk-associated factor that is sufficiently well substantiated to justify its incorporation into the model. For instance, while sufficient evidence on clinical validity with respect to mutation prevalences and disease penetrances has been established in specified risk groups, it is, in most instances, still lacking for the general population, prompting for further research in order to eventually widen risk assessment as an offer to the general population. At this given time, therefore, risk-adjusted screenings are only feasible for well-studied risk groups, such as high-risk families according to validated anamnestic criteria [35].

Finally, end points can then be collected by amalgamation with, e.g., existing national registries, and other stud- 
ies. Routinely collecting outcome data could also allow the development of digital systems which continuously generate more evidence on the clinical utility of risk assessment using these tools, increasing accuracy with increasing amounts of data drawn from rolling this learning screening system out to the general population, and paving the way to integrating evidence-based risk factor assessments into routine clinical practice in a public screening program.

\section{Prerequisites for Justified Screening}

The term "screening" seems to have become the subject of a relatively wide and, accordingly, diverging use in the field. For example, it seems that various practical experiences with the implementation of screening measures in the past have led to many political and societal discussions. Rising awareness and knowledge about risks and risk prediction have done their part to modify the traditional ideas of screening. Many initiatives to personalize risk have become known as "screening" programs, although they extend the original understanding of the term used in the context of an intervention. For the purposes of the points made in this article, we define "screening" as a systematic offer of medical diagnostic procedures at group or population level to persons who are not known to the provider to have specific medical symptoms or complaints, targeted to find/exclude latent disease or risk factors for the development of disease, in the interest of the person involved.

The introduction of such a screening program requires balancing the interests of stakeholders, and assessing the potential use as well as possible harms and costs of the program. This process is commonly referred to as the justification of a particular screening program, and there has been ongoing discussion in the literature regarding the prerequisites, which need to be fulfilled to consider a program justified [7].

Important points to take into account include the relevance of screening (incidence, prevalence, burden of disease), its clinical benefit (numbers needed to screen; screening failures; interval cancers; positive and negative predictive value influence on morbidity and mortality), medical risks and harms associated with the screening (overdiagnosis, side effects, psychological burdens, etc.), and matters of equity (access to risk counselling and preventive health care, cut-off levels, ethical aspects of the "healthy ill/sick," reimbursement, and communication of risks) [7]. These reflect general trends in Western countries and medicine, i.e., a shift from paternalism towards informed decision making, the emphasis on managed care models and quality assurance, and the importance of serious genetic conditions even if they are rare. These trends also contribute to an increased role of personal utility for individuals at stake rather than overall population clinical utility $[4,5]$. The criteria are in detail:

- The screening program should respond to a recognized need,

- the objectives of screening should be defined from the outset,

- there should be a defined target population,

- there should be scientific evidence of screening program effectiveness,

- the program should integrate education, testing, clinical services, and program management,

- there should be quality assurance, with mechanisms to minimize potential risks of screening,

- the program should ensure informed choice, confidentiality, and respect for autonomy,

- the program should promote equity and access to screening for the entire target population,

- program evaluation should be planned from the outset,

- the overall benefits of screening should outweigh the harm.

For most of the mentioned criteria, risk-adjusted screening shows a number of distinctions in comparison to established screenings, which focus on a very limited risk assessment (basically, age) to open the gates for early detection. The additional value of risk-adjusted screening to determine risk profiles before putting a large number of possibly low-risk persons through early detection methods including associated psychological burdens and uncertainties associated with the detection method is an important factor for its ethical justification - since established screening programs fail to take into account the wealth of constantly evolving knowledge and its impacts on cancer risk prediction models.

Andermann et al. [13] add further considerations to the original criteria for genetic screening policy decisions. The additions reflect the iterative nature of decisionmaking and the necessary balancing of different perspectives (including individual vs. population viewpoints), comparing alternatives, considering whether implementation in a given context will allow the benefits of screening program to be realized, and emphasizing that adequate governance and regulatory frameworks are required (see below IV.5).

These criteria widely correspond to the "ACCE" model, which has been developed by the Centers of Disease Control and Prevention as early as 2004 to evaluate genetic testing through a series of 44 questions. They em-

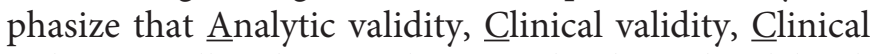
utility, as well as the compliance with other Ethical, legal, and social issues (thus the acronym ACCE, cf. CDC 2004) [36] should be a prerequisite for justified screening, and 
have also been adopted by the EuroGentest for the development of clinical utility gene cards [37].

Considering the current state of evidence and care situation, sufficient analytical and clinical validity should be a prerequisite for risk factors to be offered to be analyzed. This means specifically that analytical and clinical validity of risk factors must have been assured, while clinical utility of preventive measures taken on the basis of them can then be gathered by prospective follow-ups and outcome measures and comparison with cancer registries. Importantly, clinical validity comprises knowledge about mutation prevalence in the respective screening group as well as age-specific disease penetrances of risk factor-positive subgroups. In turn, only criteria can be included that have been validated at least in prospective cohort studies. Other factors which have not been identified or which have not yet shown to be statistically relevant will continue to be assessed by classic methods of clinical trials and research and can, once proven to be of significance, be introduced into risk assessment of the risk-adjusted screening.

In structured and reimbursed clinical care programs, therefore, only such factors should be analyzed and their results communicated.

The clinical utility of an investigation of risk factors further includes evidence that, in the event of a positive test result, efficient clinical measures are available to reduce the risk of disease or improve prognosis, and that there is, overall, proof that the investigation of a risk factor brings about a positive effect in the endpoint of clinical care.

This pertains to one of the major prerequisites for a screening as defined by Wilson and Jungner above: it is the demand for scientific evidence of screening program effectiveness. As outlined, evidence about risk factors' influence on disease development as such is readily available for many of them, and, naturally, only these factors should be incorporated into a model for risk-adjusted screening. However, the evidence regarding the overall utility of riskadjusted screening has not been comprehensively addressed. In practice, this is mostly hindered both by an ever-increasing and constantly changing knowledge about risk factors and their interdependencies, but also by an increasing amount of stratification and ever-smaller subgroups of individual sets of risk factors.

Nevertheless, it remains highly doubtful that newly available and ever-increasing knowledge about further, especially genetic, risk factors, should be held back from the population while waiting for evidence regarding clinical utility of a risk factor model which will only be outdated by the end of the studies. It seems also unlikely that factors which are known to be of analytical and clinical validity and thereby suited to assessing persons' risk to develop a disease should turn out to be of no effect for improving to target the correct persons at risk for screen- ing within a risk-adjusted screening program - which can and should, from the outset, complement existing screenings.

Rather, if no comprehensive risk assessment is offered by established clinical care paths, especially the use of privately offered Direct-to-Consumer genetic tests will likely increase due to a rising public awareness of genetic risk factors for cancer. However, in many of these tests for genetic risk factors, genetic analyses are performed without reliable knowledge of their disease association. These tests should therefore be rejected in clinical care as they may lead to uncertainty and the risk of unnecessary follow-up tests. Apart from the challenge to safeguard their quality and the correct interpretation to consumers, this would also hinder the generation evidence, as results from these tests' use will mostly be scattered among different providers and held in private databases, precluding an integrated evaluation of the used risk factors overall.

For these reasons, we propose that instead of providing screening measures only on the basis of already established evidence about the large-scale outcomes of the specific risk model as a prerequisite, a clear concept for the generation of scientific evidence for a risk-adjusted screening model over its lifetime and strict ongoing evaluation should be required for such a risk-adjusted screening, which constantly generates evidence about the model as such, the included risk factors, and multifactorial interdependencies, and which integrates new knowledge over time as it becomes available and proven. In the end, by not withholding newly available knowledge from its integration into care on the grounds of year-long evaluation of the long-term utility of different risk factors, and establishing comprehensive measures for scientific evidence and quality assurance during their use, scientific standards can be safeguarded much more quickly, effectively, and permanently. After all, since the aim of a screening program is to benefit a population of people at risk of developing a severe disease, a multi-step and self-learning screening process of risk identification alongside safeguarding scientific standards, and the continuous update of reliable evidence for risk factors, should as such be an ethical requirement.

\section{Specific Challenges and Chances of Risk-Adjusted Screening}

\section{Risk Assessment}

One of the major challenges lies in the determination of individual risks. As outlined before, the current genetic landscape of breast cancer is complex, with over 300 confidently assigned rare and common risk genes and ge- 
netic variants that are associated with high, moderate or small increases in relative risk compared to the population average. These genes and alleles act in a multiplicative manner with each other and non-genetic risk factors. It has become clear that simple Mendelian monogenic traits, in which a limited number of discrete phenotypic outcomes are due to a single gene variant, are an exception rather than the rule.

A number of genetic models to calculate absolute breast cancer risks based on gene test results are available and are continuously being updated with new information. One of the most comprehensive ones is the Breast and Ovarian Analysis of Disease Incidence and Carrier Estimation Algorithm (BOADICEA) [38], an online, CEmarked tool in which information on risk factors can be uploaded to calculate an integrated single risk score for breast and ovarian cancer. Presently, this information includes genetic data (test results of BRCA1, BRCA2, ATM, CHEK2, PALB2, and an SNP profile), family history, hormonal risk factors, and breast density, among others. The model specifies, in a quantitative way, how these various risk factors interact. It has been validated in a number of prospective breast cancer cohorts and shows superior calibration relative to other existing models. Since its discriminative power has been established in detail, it can be used to inform risk-adjusted screening approaches in the general population. In order to point out the particularities of genetic and non-genetic factors and their role in the manifestation of disease, breast cancer serves as an example for the general thoughts and arguments on riskadjusted screening as it has most thoroughly been examined for the classical screening criteria as well as genetic background.

\section{a) Genetic Risk Factors}

After the discovery of the high-risk genes $B R C A 1$ and $B R C A 2$, many countries have introduced gene carrier detection and prevention programs with the aim of reducing disease burden by risk-reducing surgery and improving disease survival by early detection. Published results indicate that these measures are effective with regard to reduced disease penetrance and the detection of earlystage tumors although data on hard endpoints are still largely missing due to limited follow-up or study time [39-43]. The spectrum and the frequency of gene mutations in particular populations are different, and the strategy for genetic testing should take into consideration the presence of frequent founder mutations. Cost-effectiveness may also be a factor in choosing testing strategies in specific populations.

Recent advances in nucleotide sequencing techniques allow the analysis of unprecedented high numbers of cases and controls, leading to the discovery of additional risk genes and alleles and underlining the genetically hetero- geneous nature of breast cancer. Over the next decade, this trend is expected to make whole genome data on large numbers of population-based subjects accessible for genetic research, which will eventually completely explain the missing heritability and familial relative risk. Presently, many commercial companies are offering gene panel testing for the prediction of breast cancer risk, comprising all genes for which there is some evidence of association with breast cancer [44]. However, according to the proposed ACCE model, only analytical validity, i.e., the accuracy with which a test detects the presence of a mutation, has been sufficiently evaluated for these tests. Data on clinical validity, i.e., age-specific associations of mutations with disease risks, and clinical utility, i.e., the outcome of preventive measures based on the genetic test results, are largely missing.

Moreover, the breast cancer risks associated with typical rare genetic defects such as those in BRCA1 and BRCA2, can be further modulated by common genetic variation [45] as well as non-genetic risk factors [46]. Validation in large population-specific prospective cohorts is largely pending. The combined effect can be calculated as a polygenic risk score (PRS) by risk prediction models, such as BOADICEA, a tool that is constantly extended and improved by ongoing studies such as the HORIOZON2020 funded BRIDGES (PI Peter Devilee) and B-CAST (PI Marjanka Schmidt) studies, and the Genome-Canada funded PERSPECTIVE study (PI Jacque Simard) for the identification and validation of risk genes for breast cancer.

Online supplementary Table 1 (for all online suppl. material, see www.karger.com/doi/10.1159/000517182) summarizes currently known genetic risk factors for which a significantly increased risk for breast cancer has been demonstrated. They are therefore considered to require clinical interventions although their clinical validity with respect to age-specific disease risks and their clinical utility with respect to morbidity and mortality reduction based on the uptake of preventive measures is not sufficiently proven yet.

\section{b) Non-Genetic Risk Factors}

For sporadic breast cancer, various non-genetic risk factors have been identified with varying levels of evidence, including lifestyle, hormonal, and biological factors. Online supplementary Table 2 summarizes the major non-genetic risk factors with strong evidence from prospective cohort studies as the Million Women Study and meta-analyses. Mammographic density and hormone replacement therapy confer relative risks of greater than two, whereas the other risk factors remain below a relative risk of 1.5. The factors listed in online supplementary Table 2 have recently been incorporated in the comprehensive risk prediction model BOADICEA [38]. 
c) Determination of Genetic and Non-Genetic Risk

Factors and Their Interaction

As outlined above, a small number of women are genetically predisposed to high risks of disease, but all women will have a certain distribution of the common low-risk variants which might modify their risk in either direction away from the population average. It has been estimated that the lifetime risk of overall breast cancer for women in the top 1 percentile of PRS alone (i.e., in the absence of high- or moderate-risk alleles) is $32.6 \%$ [47]. In addition, recent studies indicate that lifestyle may also contribute to the disease penetrance. In medicine, lifestyle is defined by specific behaviors of an individual, thus constituting non-genetic risk factors. They can be influenced by or interact with genetic factors. Even metabolism of external hormones, food, or alcohol depends on the genetic composition of an individual, thereby underlining the complex nature of carcinogenesis. Gene-environment association studies are therefore important and will eventually clarify the degree of genetic determination for each of these factors. Recently the BOADICEA comprehensive risk assessment tool has therefore incorporated major non-genetic risk factors by an interaction model that allows including these factors into risk stratification. Importantly, this model needs prospective validation, calibration, and customization in different countries and populations [38]. This can be achieved by large-scale prospective cohort studies preferably undertaken within international collaborations. The breast cancer association consortium (BCAC) and the consortium of investigators of modifiers of BRCA1/2 (CIMBA) represent excellent demonstrators that and how this can be achieved. Integrating such prospective cohorts into clinical care by the proposed cross-sectoral networks with outcome measures enabled by companion registries will allow genomic medicine to be integrated and evaluated in a non-disruptive manner in conventional medicine and will provide everyone with a structured, equitable, and transparent access.

\section{d) Conclusion}

In conclusion, one of the biggest challenges for individual risk profiling is to determine which risk factors are to be included into the risk assessment under circumstances that either preclude or hamper collecting clinical evidence. However, this task is not impossible - validating the risk prediction algorithm and defining cut-off points for the offer of either screening or irreversible and life-altering preventive measures such as mastectomies, are essential prerequisites.

As an example, the BOADICEA risk calculation algorithm, which incorporates data from multiple case control and cohort studies, has recently been validated in sev- eral prospective cohort studies of different populations for its predictive power by comparing expected to observed incidence rates in the general population as well as in risk groups for familial breast cancer [48-50] (personal communication by the group of Doug Easton, Cambridge and presentations at BRIDGES Online Closing Symposium: Breast Cancer Risk and Prognostication: Germline and Tumor Genetics, February 23-24, 2021). Although BOADICEA is now ready for clinical use with risk predictions valid for both the general population and at-risk groups, implementation still requires manifold conceptual decisions, for example, on the definition of target groups, entry points, and threshold levels for the offer of preventive measures and adequate communication strategies.

Therefore, a clear and pragmatic procedure for collecting robust outcome measures in an appropriate clinical setting will also be necessary. While more and more risk factors become known, and multi-gene panel testing will continue to include more genes, a strategy must be developed in how far and in what way this new knowledge and newly available testing can be integrated into a learning risk-adjusted screening program. Since there is always a lack of prospective evidence for newly identified risk factors with respect to the predictive values from genetic testing, genotype-specific penetrance, spectrum of phenotypes, and efficacy of interventions in populations [51], gaining reliable prospective evidence for risk assessment and the efficacy of preventive measures in genetically defined subtypes is of prior importance.

Calibrating risk prediction models and risk-adjusted prevention based on them requires sufficient data. However, for small subgroups of cancer types, a much larger overall cancer group would be required as well as sufficient data about the cancer type to subgroup the patients. Patient choice (especially around risk-reducing surgery) will impact some outcome measures but provided all interventions are reliably captured, these would feed into economic modelling and overall survival data to offer the most robust primary end point. As prospective randomized clinical trials are in general not practical under these circumstances, systematic longitudinal investigations in large populations with full genetic information available allow estimates of disease penetrance and clinical disease course (cf. the UK Biobank Study, PMID: 30305743 [52] or the registry of the German consortium for Hereditary Breast and Ovarian Cancer [52]). Therefore, patient-related documentation of large prospective cohort studies offers the ability to evaluate relevant patient outcomes and is a powerful tool to generate evidence. Importantly, interpreting patient data requires checks of internal validity and sometimes the use of external data sources to validate key assump- 
tions. As a prerequisite, entrance criteria based on now available valid and reliable risk assessments need to be determined.

\section{Risk Communication and Perception}

One of the most important aspects of any screening program is that those who are being offered screening should be fully informed about the risks and benefits so that they can give a fully informed consent. Accordingly, the communication of risk levels and the understanding by the affected person are of vital importance to meet the goal of screening programs. In particular, medical decisions depend both on the benefits and risks of interventions as well as on individual preferences and values of persons affected. In the end, a decision is up to the affected person, not the physician: any person is free to decide whether to undergo any medical intervention and even whether he or she wants to know about their individual risk levels. While recent studies suggest [53] that a majority of $78 \%$ of potentially affected persons wanted to know their risk, $13 \%$ were uncertain and $9 \%$ declined to find out. This may be a fraction of the overall population at risk but a major aspect of personal freedom to be respected.

In order to freely decide to undergo an intervention, the person needs to be provided with true, understandable, and comprehensive information about it. This requires that both affected persons and health professionals understand the risks and benefits of available medical options (such as screening), which, in turn, requires comprehensive risk communication adapted to the individual risk and health literacy level of the affected person. However, risk literacy in health care is often wanting, and most doctors and patients do not understand the available medical evidence, especially because mostly relative risks instead of absolute ones are being communicated [54]. Personalized risk communication to ensure patient autonomy and informed consent is therefore challenging, yet a recent Cochrane review suggests that receiving personalized risk information yields better understanding and more informed choices than receiving general risk information [55]. The risk estimates which need to be communicated can be worked out in a straightforward manner by combining with population incidence rates and pointing out the complexity of risk predictions in light of the immense and growing variety of risk factors.

Raising overall health and risk literacy levels in affected persons (and physicians) calls for a societal process. Risk communication can already be much improved by representing the information more effectively so that a person with low health literacy can also understand it. There is a vast amount of literature identifying methods of effective communication $[56,57]$.
The most important recommendations are to use absolute rather than relative risks, to clearly specify the reference class (i.e., the denominator) and the time frame, to use natural frequencies rather than conditional probabilities, and to communicate mortality rather than survival rates. Fact boxes are an example of a successful representation that utilizes all of these principles. They are simple tabular representations of the benefits and harms of particular treatments and have been developed and tested with laypeople, e.g., by Schwartz et al. [58]. Visual formats such as icon arrays are also a promising way to represent clinical evidence effectively. Most people prefer visual formats over numerical information [59], and particularly people with difficulties to understand numerical information (i.e., low numeracy) may benefit from them [60]. In this regard, it is important to communicate risks in manageable time units, e.g., 10-year periods. Lifetime risks are less relevant for the individual and will generally be misunderstood because they quantify risk from birth and do not match the actual risk at a given age. The communication of residual lifetime risk is also subject to misinterpretation or significant uncertainty, because it does not indicate at what point this residual risk manifests itself and with what probability. More specifically, visual formats help to reduce judgment bias such as the ratio bias $[61,62]$, framing effects [63], and the undue influence of anecdotes [64]. An example is shown in online supplementary Figure 1, which visualizes the absolute disease risks for $B R C A 1$ mutation carriers in 10-year intervals in relation to 100 individuals. There is some indication that visual formats may be particularly helpful to convey the essential aspects of the information, whereas numerical representations are better to convey more precise aspects [65]. Of course, risk communication should not be limited to risk information but should also consider psychosocial and emotional elements [66, 67].

\section{Perspective of Persons at Risk}

Although great advances in medicine are turning cancer more and more from a deadly into a curable or chronic illness, cancer is still among the most feared diseases. Thus, early detection and preventive measures to lower the risk of cancer development are of very high interest. However, risk-adjusted cancer screening is a very complex issue as its prerequisites and outcomes concern various aspects of an affected person's life and may also affect the life of related family members.

Before discussing screening details, one important aspect that matters in the discussion about risk-adjusted cancer screening concerns the affected person's fear. Screened persons may not necessarily be informed about cancer, especially about current preventive and therapeutic chances, their limitations, and survival rates. The 
screening for and determination of risk factors may pose psychological burden of unknown threat to affected persons. People may learn about an elevated cancer risk they never connected to themselves. Therefore, it is of utmost importance to provide information and counseling adapted to the people's needs and level of knowledge at every step during the screening process (also cf. infra IV.4).

Risk communication should be performed in a responsible and comprehensible way and information material presented in plain language and, if feasible, with visualizations. It should explain:

- magnitude and quality of risk assessment

- disease penetrance regarding manageable time frames (as outlined, e.g., in online suppl. Fig. 1)

- scope of consequences of the particular risk, including effectiveness and side effects, contributing and competing risks

- implications for caretakers, close others, and family

- consequences regarding insurances or future financial plans.

In case risk assessment is performed by genetic testing, a thorough counseling concerning predictive genetic testing by an approved physician and time for consideration are important (cf. infra IV.4). The right not to know must be clearly communicated and applied if desired. As knowledge about a genetic predisposition to cancer may lead to insecurities and anxiety, patients should, as part of the information process, have access to psycho-oncologists and be informed about specific selfhelp groups.

Measures for early detection must be stratified according to the risk factors. Patients must be monitored close enough to prevent interval events, but loose enough so that checkups are not present in the patient's life for most of the time. The monitoring process must be as convenient as possible, psychological burdens from it must be addressed, e.g., by patient reported outcome measures (PROM).

In this respect, patients may consider surrogate factors as equally important outcomes, such as availability of less intensive treatment options in case of early diagnosis.

In summary, since risk-adjusted cancer screening is addressed to persons at risk but nevertheless healthy individuals, the medical ethos primum non nocere, secundum cavere, tertium sanare should be met at every step.

\section{Ethical and Legal Requirements}

The implementation of screening measures also requires meeting legal, ethical, and social prerequisites. Firstly, the legal framework must allow for the implementation of a certain screening. These aspects range from specific regulations regarding informational autonomy, consent into information processing, rules on whether individuals may be contacted in order to participate in a screening, on how they can be motivated to participate, under what circumstances they can refuse to participate, as well as aspects of reimbursement for the measures by statutory health insurances and so forth. Secondly, an important social aspect is that the population needs to be able to accept a screening to be introduced as "sensible." Persons at risk must be willing to participate on the grounds of an advantage to them: it seems natural that the higher the acceptability of a screening measure is and can be communicated to the population the higher the probability of participation and successful screening. Vice versa, it is of vital importance to make the public aware of the advantages of such a screening by streamlined information rather than to concentrate on the mere legal obligation or motivation. Thirdly, ethical requirements must be met.

In particular, one of the most important ethical issues is the autonomy of the person to be screened. Informed consent of an individual to participation in screening is universally, both legally and ethically, required (Article 3 of the European Charter of Fundamental Rights and specific national rules in the respective member states' jurisdictions; cf. also [68]). This means in turn that the individual must be able to choose for oneself whether to undergo risk-adjusted screening and potential subsequent treatment. Firstly, to guarantee the autonomy of the person and ensure informed consent requires that people to be screened understand why and how their risk is elevated (cf. supra IV.3). Secondly, they need to understand potential consequences and their impact. Potential consequences include the need for further testing, which informs whether there is an elevation in the first place and how high it is. Importantly, people also need to know that testing (particularly genetic testing) can have implications for their relatives. Finally, people need to know about the benefits and harms of preventive measures that would be available if it turns out that their risk is elevated, and how these benefits and harms differ depending on the risk elevation. Importantly, they need to know about the whole chain of potential consequences before even making the first decision, as, for instance, deciding about whether to get genetic tests has to be considered in light of the options that are available given different test results.

If prediction is based on genetic research or analysis, genetic counselling must generally also be provided by a qualified person, discussing the possible medical, psychological, and social questions in connection with the performance or non-performance of the genetic examination and its existing or possible examination results. While national laws differ within Europe, EU treatise [69] provides a common frame of reference, also with regard to the admissibility of genetic screening programs for 
health purposes in general. From a practical viewpoint, as genetic testing becomes more and more available and can also increasingly take its role in health care, strategies will foreseeably be necessary to address the growing need of comprehensive and high-quality counseling for the persons considering to undergo genetic testing. Discussions have already ensued regarding the intensity of counseling necessary for undergoing polygenic risk score assessment versus testing for high-penetrance genes. There may also be adjustments in the regulatory setting, e.g., on how to deal with incidental findings of other disease risks, and on possible obligations for affected persons to share findings of genetic testing with insurance companies and employers including adverse consequences deriving form testing in the long run.

Consent must also be gained regarding the collection of data, including the possibility of re-contact, and the particular use of the data, also in case it is to be used for scientific purposes. Local jurisdiction may impose a duty to share certain information, if it is of especially high value for the population as a whole, but regulation varies from country to country (cf., for the European framework, the General Data Protection Regulation [GDPR] and, in particular, Art. 49 para. 1 lit. g and recital 157 [70]). In addition, it needs to be considered how to deal with incidental or secondary findings. Reciprocally to the right to opt out of a screening program, different health care systems can also offer possibilities to increase motivation of individuals to take part in screening programs. Accordingly, both legally and ethically, the implications for the use of collected genetic data by screening must be taken into account: Especially, when samples are stored for future use and could be interposed with additional data to be gathered later, the ownership of samples, data, and results is of the essence. Moreover, a secondary use of the resulting risk profiles could result in discrimination by third parties, e.g., insurance companies or employers.

In addition, statutory health care regimes should be updated to allow addressing certain disease risks rather than manifest disease only. This phenomenon has become known as the problem of the "healthy sick" - denoting persons currently without symptoms but with a high risk of developing a severe disease over time which could be avoided by early diagnosis and therapy. As many social systems have high burdens for including new health care measures into their schemes of health care provision [71], it is of essence to identify what treatments and diagnostic measures can be particularly helpful for avoiding manifest disease in the "healthy sick." These can also contribute to cost-effectiveness, as high treatment costs for manifest disease can be avoided by much lower costs for earlier measures whenever a specific risk justifies early diagnosis. The more elaborate the knowledge about specific risks of disease will become due to advancing in- sights into genetic and other risk factors even before a disease manifests itself, the more important it will be to address the issue of prevention as a part of an integrative rather than merely curative health care scheme, and to define specific measures which are covered within its scope [72].

Finally, the prerequisites for implementation of a certain screening program in a given country must allow for the particular design of the screening. Legal but also sociocultural and ethical rules can be quite different in various jurisdictions (cf., for cervical cancer, an overview of current legal frameworks in [73]). Regarding consent and data protection, the GDPR provides harmonized protection within the jurisdictions of and across the EU. However, prerequisites for an internationally accepted riskadjusted screening program, which is also financially accounted for in different health care systems, and the offer of a standardized high level of risk assessment, early detection, and treatment across national boards of program and strategy assessment will remain a goal for further international harmonization.

\section{Call for Action}

The constant gain of knowledge about genetic and non-genetic risk factors must be considered and incorporated into clinical practice rather than ignoring newly gained knowledge. While best quality evidence must continue to be sought, alternatives to RCTs will take short-term advantage of modern technologies whilst continuing to embrace the wider principles set for a public screening program. Ultimately, existing screening programs should be assessed to evaluate whether they can be adapted to accommodate an institutionalized multi-step risk-adjusted learning screening system, which transcends existing approach to screening largely using age and family history to stratify risk (cf., regarding effectiveness of risk-based vs. age-based screening, [74]). Persons developing the disease screened for should be offered genetic and pertinent non-genetic assessment, and collected data should be fed into a learning screening system.

Entry points for screening should be defined according to the state of current knowledge of risk factors and models, stratified by risk groups. Relatives of affected individuals may be the first to be offered the risk-adapted screening program. This system should be constantly evaluated regarding forthcoming insight into new genetic and other risk factors, allowing the application of stratified screening strategies, and continuously updating genetic risk assessment tools within a clinical setting. Eventually, this learning screening system can be rolled out to younger women who may be carriers of genetic muta- 
tions as well as, ultimately, more general parts of the population, once evidence on its clinical utility has been established in practice.

On the grounds of the findings laid out above, we believe that the following steps should be taken to better target breast cancer and comparable health risks, and to ease the necessary transition from a retrospective approach of early detection screening towards a wider, earlier, and more streamlined approach of risk-adjusted prediction, prevention, and disease management.

\section{a. Fostering Prospective Outcome Evaluation: Tumor}

Registries Complemented by Genetic and Preventive

Information

Prospective cohort studies on the effectiveness of preventive measures based on validated risk factors and documented within registries will allow medical outcome measures as a prerequisite for the transition from age- to risk-adjusted screening. Several nationwide registries already exist that can be harmonized and merged. Activities supported by the EU such as the ERN Genturis project [75] are already ongoing in order to establish a reference network and define a meta-registry for a pan-European development in order to harmonize patient registries and health care pathways. For example, an important outcome parameter to monitor during the implementation of risk-adjusted screening is whether the proportion of detected invasive disease remains the same, while that of overdiagnosis declines. Outcome measures should also be assessed as to whether they are not only medically determined but also patient relevant. An accompanying data protection concept addressing relevant ELSI issues that has already been compiled can serve as a paradigm for different familial tumor syndromes.

\section{b. Research}

In order to justify making risk-adapted screening decisions on the grounds of specific risk factors, these factors need to be sufficiently substantiated by a minimum standard of evidence regarding their clinical validity. For instance, mutation prevalences and disease penetrances have been well established for specified risk groups, proving their relation to the risk of disease development. However, in most instances, such evidence is still lacking for the general population, prompting for further research on risk factors for other groups than identified high-risk groups.

Also, the sensitivity of specific screening modalities depends on histology and genetic make-up. For instance, for a group of high-risk women with dense breast tissue the sensitivity of a mammogram is not sufficient. Therefore, additional imaging procedures such as tomosynthesis and MRI need to be further explored in those subgroups.
Beyond medical utility and evidence, further investigation is required regarding the public health outcomes of implementing risk-adjusted screening in health care systems: while we assume that preventing disease instead of treating it will save costs rather than increase them, and, even so, while preemptively avoiding disease development in a person should also have a value of its own, the economic impact of risk-adjusted versus age-based screening should be modelled and evaluated as risk-adjusted screening becomes available from the onset, in order to gain health economic knowledge for policy decisions which will be difficult to gather at a later point in time. Generally, these and other pressing research needs should be addressed by a dedicated research strategy for funding and coordinated on a high level, such as national, European, and international research programs and institutions.

\section{c. Strengthening Knowledge/Evidence-Generating Networks}

Inter- and transdisciplinary networks need to be strengthened and widened in order to address the specific needs to implement new knowledge into routine clinical work, allowing access to screening services and risk assessment and make a low-threshold offer to a wide public. These services need to be fostered by educational programs constantly disseminating the generated evidence and increasing knowledge on genomic medicine with health care professionals and the general public, mainstreaming and keeping up to date the state of knowledge in clinical care. Hospitals and health care providers should come up with a concept how to incentivize and implement this approach, e.g., by special contracts and reimbursement with statutory sickness funds. The German consortium is currently providing such an approach and could already build up a trans-sectorial network capable of providing nationwide support.

\section{d. Further Development of Checklists for the Identification of Target Groups}

Easy-to-use checklists and guidelines proved their worth for the identification of target groups, i.e., groups of persons at potentially higher risk, which can be identified more easily by the use of such checklists. They can be adapted to different situations according to the addressee, e.g., for health care professionals in practice, for patients and relatives as self-assessment, and so forth. The use of an evidence-based, up-to-date, and comprehensive version of a checklist should be a compulsory requirement in certified cancer centers. As an example, the German Cancer Society stipulates the use of a validated checklist for the identification of persons at risk for breast cancer in certified breast cancer centers $[3,32,76]$. 


\section{e. Improving Risk and Genetic Literacy of Counselors and Counselees}

A prerequisite for appropriate risk assessment and communication is the competence of health professionals in this field who will, in practice, serve as risk counsellors for the affected persons. However, the steep acceleration of knowledge gain in genomic medicine and risk calculation along with its hasty introduction into clinical diagnostics makes it nearly impossible for health care providers to either effectively deliver or prevent the development. Therefore, additional competencies need to be acquired preferentially within structured and evidencebased educational programs to guide clinicians [27, 52]. The improvement of risk and genetic literacy both for counselors and counselees is a prerequisite for autonomous decision-making of the persons at stake, as well as the uptake of risk-adjusted preventive measures. Specific training should be offered as well as specified and up-todate patient decision aids based on the currently best available evidence.

With the introduction of gene panel testing classification of genetic variants has become a major challenge. Conjoint international activities such as the ENIGMA consortium and the BRCA challenge aim to build up knowledge bases in order to continuously improve clinical interpretation and decision-making. The incorporation of genetic specialists into interdisciplinary clinical tumor boards would further promote genetic competence of clinical practitioners.

Also, decision coaching by specialized nurses could further support genetic counseling. Moreover, innovative web-based resources such as the Public Health Genomics Knowledge Base (PHGKB) of the CDC may support a continuous learning process and connect populationbased research with public health applications on clinical genomics [77].

\section{f. Validated Risk Prediction Models}

Reliable risk prediction is crucial and risk determination programs such as BOADICEA need to be further developed, as is the case within the EU Horizon 2020 funded BRIDGES project. According to the new medical product law, risk models need to be certified and validated (notwithstanding clinical validation as called for by the ACCE requirement, cf. above), which is best achieved within knowledge-generating networks of care. Networks of expert research centers, cancer centers, and primary care practitioners should also jointly deliver digitized risk estimations and risk-adjusted preventive measures based on risk factor-driven, quality-assured, and adaptable risk prediction models, and define common entry points for administering such risk assessment, e.g., on the occasion of existing health screening programs for the general population, on the basis of disease prevalence (e.g., cf. [12]).
The existing knowledge and new findings about risk factors regarding different risk groups should be made available for policy makers and health professionals in prediction and screening guidelines.

\section{g. Data Safety and Ownership}

In addition to the considerations above (cf. IV.5), collected data and test results, especially when interpolated with other existing data, should be ensured to remain with the public domain in the long run. They should not be shared with or passed on to commercial interests for economic purposes or reasons other than disease control and public health for which the data were collected.

Given these prerequisites, we believe that cancer screening should finally be moving forward from an agebased primary early disease detection towards an integrated, multi-step, and evidence-based risk-adapted approach in which individual risk assessment would allow a much more precise way of preventing disease for persons at high risk while at the same time saving both cost and adverse outcomes for low-risk persons. Instead of one-size-fits-all early disease detection programs leading to therapy only when a disease is already manifest, science, medicine, and politics should work together to offer high-quality and evidence-based individualized prevention programs, or people will resort to privately offered alternatives which can be of varying quality, profit-driven, not centrally evaluated, and with uncertain outcomes. While medicine continues towards becoming increasingly individualized both in diagnosis and therapy, screening and disease prevention should, while assuring representation, justification, and evaluation, follow and make good use of the new possibilities medical knowledge has to offer.

\section{Key Message}

Risk-adjusted prevention based on established risk factors should be offered in the context of knowledge-generating care.

\section{Acknowledgement}

This paper was compiled between 2015 and 2020 by an international expert group in collaboration with the Joint Action Initiative CANCON (Cancer Control Joint Action) and the subsequent joint action iPAAC (innovative Partnership for Action Against Cancer) in order to explore innovative approaches to cancer prevention and control.

\section{Conflict of Interest Statement}

The authors have no conflicts of interest to declare. 


\section{Funding Sources}

The project was funded by the German Federal Ministry of Health (grant No. 2515FSB401 to Rita Schmutzler and Christiane Woopen) for supporting the international expert meetings, and a grant of the EU Horizon 2020 program, BRIDGES (grant No. 634935, PI Peter Devilee, WP5-PI Rita Schmutzler), for the compilation of the most recent findings of genetic risk prediction.

\section{Author Contributions}

Rita Schmutzler and Björn Schmitz-Luhn wrote the manuscript. All authors were involved in project conception and the development of the papers' arguments. All authors read and approved the final manuscript.

\section{References}

1 Lichtenstein P, Holm NV, Verkasalo PK, Iliadou A, Kaprio J, Koskenvuo M, et al. Environmental and heritable factors in the causation of cancer--analyses of cohorts of twins from Sweden, Denmark, and Finland. N Engl J Med. 2000 Jul 13;343(2):78-85.

2 Mucci LA, Hjelmborg JB, Harris JR, Czene K, Havelick DJ, Scheike T, et al. Familial Risk and Heritability of Cancer Among Twins in Nordic Countries. JAMA. 2016 Jan 5;315(1): 68-76.

3 Rhiem K, Bücker-Nott HJ, Hellmich $M$, Fischer H, Ataseven B, Dittmer-Grabowski C, et al. Benchmarking of a checklist for the identification of familial risk for breast and ovarian cancers in a prospective cohort. Breast J. 2019 May;25(3):455-60.

4 Andermann A, Blancquaert I, Déry V. Genetic screening: a conceptual framework for programmes and policy-making. J Health Serv Res Policy. 2010 Apr;15(2):90-7.

5 Brand A, Lal JA, Public Health Genomics European N. European Best Practice Guidelines for Quality Assurance, Provision and Use of Genome-based Information and Technologies: the 2012 Declaration of Rome. Drug Metabol Drug Interact. 2012;27(3):177-82.

6 Burton H, Chowdhury S, Dent T, Hall A, Pashayan N, Pharoah P. Public health implications from COGS and potential for risk stratification and screening. Nat Genet. 2013 Apr; 45(4):349-51.

7 Wilson JMG, Jungner G. Principles and practice of screening for disease. In: Geneva WHO, editor. PUBLIC HEALTH PAPERS. Geneva 1968

8 Kuchenbaecker KB, Hopper JL, Barnes DR, Phillips KA, Mooij TM, Roos-Blom MJ, et al. Risks of Breast, Ovarian, and Contralateral Breast Cancer for BRCA1 and BRCA2 Mutation Carriers. JAMA. 2017 Jun 20;317(23):2402-16.

9 Khoury MJ, Janssens AC, Ransohoff DF. How can polygenic inheritance be used in population screening for common diseases? Genet Med. 2013 Jun;15(6):437-43.

10 Pashayan N, Hall A, Chowdhury S, Dent T, Pharoah PD, Burton H. Public health genomics and personalized prevention: lessons from the COGS project. J Intern Med. 2013 Nov; 274(5):451-6.

11 Marcus PM, Freedman AN, Khoury MJ. Targeted Cancer Screening in Average-Risk Individuals. Am J Prev Med. 2015 Nov;49(5):76571.

12 Mukama T, Kharazmi E, Xing X, Sundquist K, Sundquist J, Brenner H, et al. Risk-Adapted Starting Age of Screening for Relatives of $\mathrm{Pa}$ tients With Breast Cancer. JAMA Oncology. 2019 Nov 14
13 Andermann A, Blancquaert I, Beauchamp S, Déry V. Revisiting Wilson and Jungner in the genomic age: a review of screening criteria over the past 40 years. Bull World Health Organ. 2008 Apr;86(4):317-9.

14 Schmutzler RK, Dietz D, Jöckel KH, Greinert R, Hoffmann W, Riemann J, et al. Risk-adjusted Cancer-Screening; 2011 [access: February 3, 2021]. Available from: https://www. bundesgesundheitsministerium.de/fileadmin/Dateien/3_Downloads/N/Nationaler_ Krebsplan/Risk_adjusted_screening_for_ cancer.pdf.

15 Dent T, Jbilou J, Rafi I, Segnan N, Törnberg S, Chowdhury S, et al. Stratified cancer screening: the practicalities of implementation. Public Health Genomics. 2013;16(3):94-9.

16 Van den Bulcke MSB, De Censi A, Decoster L, Federici A, Nowak F, Kholmanskikh O, et al. Policy Paper on Public Health Genomics in Cancer; 2017.

17 Andermann A, Blancquaert I, Beauchamp S, Costea I. Guiding policy decisions for genetic screening: developing a systematic and transparent approach. Public Health Genomics. 2011;14(1):9-16

18 Teutsch SM, Fielding JE, Khoury MJ, Evans JP. Utility before business. Genet Med. 2014 Dec;16(12):869-70.

19 Hoxhaj I, Stojanovic J, Sassano M, Acampora A, Boccia S. A review of the legislation of direct-to-consumer genetic testing in EU member states. Eur J Med Genet. 2020 Apr;63(4): 103841.

20 Andermann A, Pang T, Newton JN, Davis A, Panisset U. Evidence for Health III: Making evidence-informed decisions that integrate values and context. Health Res Policy Syst. 2016 Mar 14;14:16.

21 Pokorska-Bocci A, Kroese M, Sagoo GS, Hall A, Burton H. Personalised medicine in the UK: challenges of implementation and impact on healthcare system. Genome Med. 2014;6(4):28.

22 Olberg B, Perleth M, Busse R. The new regulation to investigate potentially beneficial diagnostic and therapeutic methods in Germany: up to international standard? Health Policy. 2014 Aug;117(2):135-45.

23 Bakker MF, de Lange SV, Pijnappel RM, Mann RM, Peeters PHM, Monninkhof EM, et al. Supplemental MRI Screening for Women with Extremely Dense Breast Tissue. N Engl J Med. 2019 Nov 28;381(22):2091-102.

24 Boccia S, Pastorino R, Ricciardi W, Ádány R, Barnhoorn F, Boffetta P, et al. How to Integrate Personalized Medicine into Prevention? Recommendations from the Personalized Prevention of Chronic Diseases (PRECeDI)
Consortium. Public Health Genomics. 2019; 22(5-6):208-14.

25 Skirton H, Lewis C, Kent A, Coviello DA Members of Eurogentest U, Committee EE. Genetic education and the challenge of genomic medicine: development of core competences to support preparation of health professionals in Europe. Eur J Hum Genet. 2010 Sep;18(9):972-7.

26 Chowdhury S, Dent T, Pashayan N, Hall A, Lyratzopoulos G, Hallowell N, et al. Incorporating genomics into breast and prostate cancer screening: assessing the implications. Genet Med. 2013 Jun;15(6):423-32.

27 Chowdhury S, Henneman L, Dent T, Hall A, Burton A, Pharoah P, et al. Do Health Professionals Need Additional Competencies for Stratified Cancer Prevention Based on Genetic Risk Profiling? J Pers Med. 2015 Jun 9;5(2): 191-212.

28 Slade I, Burton H. Preparing clinicians for genomic medicine. Postgrad Med J. 2016 Jul; 92(1089):369-71.

29 Schully SD, Khoury MJ. What is translational genomics? An expanded research agenda for improving individual and population health. Appl Transl Genom. 2014 Dec;3(4):82-3.

30 Marrone M, Schilsky RL, Liu G, Khoury MJ, Freedman AN. Opportunities for translational epidemiology: the important role of observational studies to advance precision oncology. Cancer Epidemiol Biomarkers Prev. 2015 Mar;24(3):484-9.

31 Chambers DA, Feero WG, Khoury MJ. Convergence of Implementation Science, Precision Medicine, and the Learning Health Care System: A New Model for Biomedical Research. JAMA. 2016 May 10;315(18):1941-2.

32 Kast K, Rhiem K, Wappenschmidt B, Hahnen E, Hauke J, Bluemcke B, et al. Prevalence of BRCA1/2 germline mutations in 21401 families with breast and ovarian cancer. J Med Genet. 2016 Jul;53(7):465-71.

33 Harter P, Hauke J, Heitz F, Reuss A, Kommoss S, Marmé F, et al. Prevalence of deleterious germline variants in risk genes including BRCA $1 / 2$ in consecutive ovarian cancer patients (AGO-TR-1). PLoS One. 2017;12(10): e0186043.

34 Engel C, Rhiem K, Hahnen E, Loibl S, Weber $\mathrm{KE}$, Seiler S, et al. Prevalence of pathogenic BRCA1/2 germline mutations among 802 women with unilateral triple-negative breast cancer without family cancer history. BMC Cancer. 2018 Mar 7;18(1):265.

35 Gierach GL, Choudhury PP, Garcia-Closas M. Toward Risk-Stratified Breast Cancer Screening: Considerations for Changes in Screening Guidelines. JAMA Oncology. 2019 Nov 14. 
36 Centers for Disease Control and Prevention. ACCE Model Process for Evaluating Genetic Tests. 2010 [access: February 3, 2021]. Available from: https://www.cdc.gov/Genomics/ Gtesting/Acce/Index.Htm.

37 EuroGenTest. EuroGenTest. 2011 [access: February 3, 2021]. Available from: http:// www.eurogentest.org/index.php?id $=160$.

38 Lee A, Mavaddat N, Wilcox AN, Cunningham AP, Carver T, Hartley S, et al. BOADICEA: a comprehensive breast cancer risk prediction model incorporating genetic and nongenetic risk factors. Genet Med. 2019 Jan 15.

39 Evans DG, Kesavan N, Lim Y, Gadde S, Hurley E, Massat NJ, et al. MRI breast screening in high-risk women: cancer detection and survival analysis. Breast Cancer Research and Treatment. 2014 Jun;145(3):663-72.

40 De Felice F, Marchetti C, Musella A, Palaia I, Perniola G, Musio D, et al. Bilateral risk-reduction mastectomy in BRCA1 and BRCA2 mutation carriers: a meta-analysis. Ann Surg Oncol. 2015 Sep;22(9):2876-80.

41 Heemskerk-Gerritsen BA, Rookus MA, Aalfs CM, Ausems MG, Collée JM, Jansen L, et al. Improved overall survival after contralateral risk-reducing mastectomy in BRCA1/2 mutation carriers with a history of unilateral breast cancer: a prospective analysis. Int J Cancer. 2015 Feb 1;136(3):668-77.

42 Saadatmand S, Obdeijn IM, Rutgers EJ, Oosterwijk JC, Tollenaar RA, Woldringh GH, et al. Survival benefit in women with BRCA1 mutation or familial risk in the MRI screening study (MRISC). Int J Cancer. 2015 Oct 1; 137(7):1729-38.

43 Li X, You R, Wang X, Liu C, Xu Z, Zhou J, et al. Effectiveness of Prophylactic Surgeries in BRCA1 or BRCA2 Mutation Carriers: A Meta-analysis and Systematic Review. Clin Cancer Res. 2016 Aug 1;22(15):3971-81.

44 Easton DF, Pharoah PD, Antoniou AC, Tischkowitz M, Tavtigian SV, Nathanson KL, et al. Gene-panel sequencing and the prediction of breast-cancer risk. N Engl J Med. 2015 Jun 4;372(23):2243-57.

45 Kuchenbaecker KB, McGuffog L, Barrowdale D, Lee A, Soucy P, Dennis J, et al. Evaluation of Polygenic Risk Scores for Breast and Ovarian Cancer Risk Prediction in BRCA1 and BRCA2 Mutation Carriers. J Natl Cancer Inst. 2017 Jul 1;109(7)

46 Narod SA. Modifiers of risk of hereditary breast cancer. Oncogene. 2006 Sep 25;25(43): 5832-6.

47 Mavaddat N, Michailidou K, Dennis J, Lush M, Fachal L, Lee A, et al. Polygenic Risk Scores for Prediction of Breast Cancer and Breast Cancer Subtypes. Am J Hum Genet. 2019 Jan 3;104(1):21-34

48 Lakeman IMM, Rodríguez-Girondo M, Lee A, Ruiter R, Stricker BH, Wijnant SRA, et al. Validation of the BOADICEA model and a 313-variant polygenic risk score for breast cancer risk prediction in a Dutch prospective cohort. Genet Med. 2020 Nov;22(11):180311.

49 Terry MB, Liao Y, Whittemore AS, Leoce N, Buchsbaum R, Zeinomar N, et al. 10-year per- formance of four models of breast cancer risk: a validation study. Lancet Oncol. 2019 Apr; 20(4):504-17.

50 Borde J, Ernst C, Wappenschmidt B, Niederacher D, Weber-Lassalle K, Schmidt G, et al. Performance of breast cancer polygenic risk scores in 760 female CHEK2 germline mutation carriers. J Natl Cancer Inst. 2020 Dec 29.

51 Burke W. Genetic tests: clinical validity and clinical utility. Curr Protoc Hum Genet. 2014; 81:9-8.15.1

52 Wright CF, Middleton A, Burton H, Cunningham F, Humphries SE, Hurst J, et al. Policy challenges of clinical genome sequencing. BMJ. 2013 Nov 22;347:f6845.

53 Hall AE, Chowdhury S, Hallowell N, Pashayan N, Dent $\mathrm{T}$, Pharoah $\mathrm{P}$, et al. Implementing risk-stratified screening for common cancers: a review of potential ethical, legal and social issues. J Public Health (Oxf). 2014 Jun;36(2): 285-91.

54 Wegwarth O, Gigerenzer G. The Barrier to Informed Choice in Cancer Screening: Statistical Illiteracy in Physicians and Patients. Recent Results Cancer Res. 2018;210:207-21.

55 Edwards AG, Naik G, Ahmed H, Elwyn GJ, Pickles T, Hood K, et al. Personalised risk communication for informed decision making about taking screening tests. Cochrane Database Syst Rev. 2013(2):CD001865

56 Gigerenzer G, Gaissmaier W, Kurz-Milcke E, Schwartz LM, Woloshin S. Helping Doctors and Patients Make Sense of Health Statistics. Psychol Sci Public Interest. 2007 Nov;8(2): 53-96.

57 Trevena LJ, Zikmund-Fisher BJ, Edwards A, Gaissmaier W, Galesic M, Han PK, et al. Presenting quantitative information about decision outcomes: a risk communication primer for patient decision aid developers. BMC Med Inform Decis Mak. 2013;13 Suppl 2(Suppl 2): S7.

58 Schwartz LM, Woloshin S, Welch HG. Using a drug facts box to communicate drug benefits and harms: two randomized trials. Ann Intern Med. 2009 Apr 21;150(8):516-27.

59 Gaissmaier W, Wegwarth O, Skopec D, Müller AS, Broschinski S, Politi MC. Numbers can be worth a thousand pictures: individual differences in understanding graphical and numerical representations of health-related information. Health Psychol. 2012 May; 31(3):286-96.

60 Galesic M, Garcia-Retamero R. Statistical numeracy for health: a cross-cultural comparison with probabilistic national samples. Arch Intern Med. 2010 Mar 8;170(5):462-8.

61 Garcia-Retamero R, Galesic M. Corrigendum to: "Who profits from visual aids: Overcoming challenges in people's understanding of risks" [Social Science \& Medicine, 70 (2010), 1019-1025. Soc Sci Med. 2010 Jun;70(12): 2097.

62 Garcia-Retamero R, Galesic M, Gigerenzer G. Do icon arrays help reduce denominator neglect? Med Decis Making. 2010 Nov-Dec; 30(6):672-84

63 Garcia-Retamero R, Cokely ET. Effective Communication of Risks to Young Adults:
Using Message Framing and Visual Aids to Increase Condom Use and STD Screening. J Exp Psychol Appl. 2011 09/01;17:270-87.

64 Fagerlin A, Wang C, Ubel PA. Reducing the influence of anecdotal reasoning on people's health care decisions: is a picture worth a thousand statistics? Med Decis Making. 2005 Jul-Aug;25(4):398-405.

65 Feldman-Stewart D, Kocovski N, McConnell BA, Brundage MD, Mackillop WJ. Perception of Quantitative Information for Treatment Decisions. Med Decis Making. 2000 04/01;20: 228-38.

66 Edwards A, Gray J, Clarke A, Dundon J, Elwyn $\mathrm{G}$, Gaff C, et al. Interventions to improve risk communication in clinical genetics: systematic review. Patient Educ Couns. 2008 Apr;71(1):4-25.

67 Riley BD, Culver JO, Skrzynia C, Senter LA, Peters JA, Costalas JW, et al. Essential elements of genetic cancer risk assessment, counseling, and testing: updated recommendations of the National Society of Genetic Counselors. J Genet Couns. 2012 Apr;21(2): 151-61.

68 Beauchamp T, Childress J. Principles of Biomedical Ethics. 3rd ed. New York: Oxford University Press; 1989.

69 Council of Europe. Additional Protocol to the Convention on Human Rights and Biomedicine, concerning Genetic Testing for Health Purposes. Strasbourg 2008.

70 Schulz M, Hennis-Plasschaert JA. REGULATION (EU) 2016/679 OF THE EUROPEAN PARLIAMENT AND OF THE COUNCIL. Official Journal of the European Union. 2016 27 April 2016.

71 Kirk BBSI, Chiotan C, Costongs C, Kunkel T, et al. Health Promotion and Primary Prevention in 14 European countries: a comparative overview of key policies, approaches, gaps and needs. Brussels 2015.

72 Meier F, Harney A, Rhiem K, Neumann A, Neusser S, Braun M, et al. Risikoadaptierte Prävention. Wiesbaden: Springer VS; 2018

73 Májek O, Anttila A, Arbyn M, van Veen E-B, Engesæter B, Lonnberg S. The legal framework for European cervical cancer screening programmes. European Journal of Public Health. 2018 09/27:29.

74 Pashayan N, Morris S, Gilbert FJ, Pharoah PDP. Cost-effectiveness and Benefit-toHarm Ratio of Risk-Stratified Screening for Breast Cancer: A Life-Table Model. JAMA Oncol. 2018 Nov 1;4(11):1504-10.

75 GENTURIS ERN [access: February 3, 2021]. Available from: https://www.genturis.eu/ l=eng/Home.html.

76 Deutsches Konsortium für Erblichen Brustund Eierstockkrebs ÄW-L, Deutsche Krebsgesellschaft, Deutsche Gesellschaft für Senologie. Checkliste zur Erfassung einer möglichen erblichen Belastung für Brust-und/oder Eierstockkrebs. 2019.

$77 \mathrm{Yu}$ W, Gwinn M, Dotson WD, Green RF, Clyne M, Wulf A, et al. A knowledge base for tracking the impact of genomics on population health. Genet Med. 2016 Dec;18(12): 1312-4. 